\title{
Science and Medicine
}

\author{
Ze'ev Hochberg and Jean-Claude Carel
}

\section{Small and variable}

\section{Origins and genetic diversity of pygmy hunter-gatherers from Western Central Africa}

Verdu P, Austerlitz F, Estoup A, Vitalis R, Georges M, Thery S, Froment A, Le Bomin S, Gessain A, Hombert JM, Van der Veen L, Quintana-Murci L, Bahuchet S, Heyer E

Ecoanthropology and Ethnobiology, UMR, CNRS-MNHN-Université Paris, Paris, France

verdu@mnhn.fr

Curr Biol 2009;19:312-318

Context: Central Africa is currently peopled by numerous sedentary agriculturalist populations neighboring the largest group of mobile hunter-gatherers, the Pygmies. Although archeological remains attest to Homo sapiens' presence in the Congo Basin for at least 30,000 years, the demographic history of these groups, including divergence and admixture, remains widely unknown. It is still debated whether common history or convergent adaptation to a forest environment resulted in the short stature characterizing the pygmies.

Methods: The authors genotyped 604 individuals at 28 autosomal tetranucleotide microsatellite loci in 12 non-pygmy and 9 neighboring pygmy populations.

Results: They found a high level of genetic heterogeneity among Western Central African pygmies, as well as evidence of heterogeneous levels of asymmetrical gene flow from non-pygmy to pygmy, consistent with the variable sociocultural barriers against intermarriages. Using approximate bayesian, they compared several historical scenarios. The most likely points toward a unique ancestral pygmy population that diversified 2,800 years ago, contemporarily with the Neolithic expansion of non-pygmy agriculturalists.

Conclusions: The results show that recent isolation, genetic drift, and heterogeneous admixture enabled a rapid and substantial genetic differentiation among Western Central African pygmies. Such an admixture pattern is consistent with the various sociocultural behaviors related to intermarriages between pygmy and non-pygmy.

Last year this Yearbook cited that pygmies have not evolved through positive selection for small stature. This was a by-product of selection for early onset of reproduction in the face of short survival in a hostile environment. The authors of the current article suggest that the spread of non-pygmy farmers through Western Central Africa may have driven pygmies into isolated groups, prompting their speedy evolution into distinct populations with a huge genetic diversity. Their average height can vary by up to $20 \mathrm{~cm}$.

Verdu et al. collected DNA samples from 9 pygmy and 12 nearby non-pygmy groups in Cameroon and Gabon. The genetic variation between different pygmy populations was of a similar scale to that between Europeans and Asians. The pygmy groups seem to have emerged around 2,800 years ago from a common ancestral group that likely split from other humans 54,000-90,000 years ago. The authors suggest that this coincides with the expansion of non-pygmy agriculture in the region, a process that might have driven pygmies into small, isolated hunter-gatherer groups that evolved rapidly. 


\section{Function of mitochondrial Stat3 in cellular respiration}

Wegrzyn J, Potla R, Chwae YJ, Sepuri NB, Zhang Q, Koeck T, Derecka M, Szczepanek K, Szelag M, Gornicka A, Moh A, Moghaddas S, Chen Q, Bobbili S, Cichy J, Dulak J, Baker DP, Wolfman A, Stuehr D, Hassan MO, Fu XY, Avadhani N, Drake Jl, Fawcett P, Lesnefsky EJ, Larner AC

Department of Biochemistry and Molecular Biology and Massey Cancer Center, Virginia Commonwealth University, Richmond, Va., USA

Science 2009;323:793-797

Context: Cytokines such as interleukin-6 induce tyrosine and serine phosphorylation of Stat 3 that results in activation of Stat3-responsive genes.

Aim: To provide evidence that Stat 3 is present in the mitochondria of cultured cells and primary tissues, including the liver and heart.

Results: In Stat3-/- cells, the activities of complexes I and II of the electron transport chain (ETC) were significantly decreased. We identified Stat3 mutants that selectively restored the protein's function as a transcription factor or its functions within the ETC. In mice that do not express Stat 3 in the heart, there were also selective defects in the activities of complexes I and II of the ETC.

Conclusions: These data indicate that Stat3 is required for optimal function of the ETC, which may allow it to orchestrate responses to cellular homeostasis.

We recognize the Stat 3 (signal transducers and activators of transcription) as a transcription factor that is activated in response to $\mathrm{GH}$ and other cytokines. We now learn that Stat3 has a distinct function in the mitochondria. It interacts with a component of complex I of the electron transport chain and interferons to inhibit mitochondrial function - the authors explored a role for Stat3 in mitochondria. In a subset of mouse B lymphocytes devoid of Stat3, oxidative phosphorylation was reduced, a defect attributable to the diminished activity of complexes I and II. It is tempting to speculate that GH and other cytokines use Stat3 to regulate transcription and respiration. Yet, cytokines may not be the only, or even the major, controllers of Stat3-modulated oxidation.

\section{Quixotic alright \\ Review}

\section{Tilting at quixotic trait loci: an evolutionary perspective on genetic causation}

Weiss KM

Department of Anthropology and Integrated Biosciences Genetics Program, Pennsylvania State University, University Park, Pa., USA

kenweiss@psu.edu

Genetics 2008;179:1741-1756

Recent years have seen great advances in generating and analyzing data to identify the genetic architecture of biological traits. Human disease has understandably received intense research focus, and the genes responsible for most mendelian diseases have successfully been identified. However, the same advances have shown a consistent if less satisfying pattern in which complex traits are affected by variation in large numbers of genes, most of which have individually minor or statistically elusive effects, leaving the bulk of genetic etiology unaccounted for. This pattern applies to diverse and unrelated traits, not just disease, in basically all species, and is consistent with evolutionary expectations, raising challenging questions about the best way to approach and understand biological complexity.

Complex phenotypic or disease traits are affected by variation in large numbers of genes, most of which have individually minor or statistically intangible effects. This pattern is consistent with evolutionary expectations. Yet, if diabetes or height shows a genetic component of $\sim 50 \%$ by twin studies, and the GWAS explain 3\% of the variation, that leaves the bulk of genetic etiology unaccounted for. 
The huge longitudinal biobanks being launched can be expected to do any better than they have already done. This important review suggests that the demand for increased sequence and sample size may itself be evidence that we are approaching diminishing returns. By chasing the effects most difficult to replicate, or hardest to discriminate between true and false positives, accurate risk estimation may be as small. While these efforts go on, it is time to realize time and again that we are not our gene sequence. Our DNA sequence is just part of the story, while gene expression may get us closer to understanding.

\section{Lamarckism is back here}

\section{Transgenerational rescue of a genetic defect in long-term potentiation and memory formation by juvenile enrichment}

Arai JA, Li S, Hartley DM, Feig LA

Sackler School of Biomedical Sciences and Department of Biochemistry, Tufts University School of Medicine, Boston, Mass., USA

J Neurosci 2009;29:1496-1502

Context: The idea that qualities acquired from experience can be transmitted to future offspring has long been considered incompatible with current understanding of genetics. However, the recent documentation of non-mendelian transgenerational inheritance makes such a 'lamarckian'-like phenomenon more plausible.

Results: This paper demonstrates that exposure of 15 -day-old mice to 2 weeks of an enriched environment (EE), that includes exposure to novel objects, elevated social interactions and voluntary exercise, which enhances long-term potentiation (LTP) not only in these enriched mice but also in their future offspring through early adolescence, even if the offspring never experience EE. In both generations, LTP induction is augmented by a newly appearing cAMP/p38 MAP kinase-dependent signaling cascade. Strikingly, defective LTP and contextual fear conditioning memory normally associated with ras-grf knock-out mice are both masked in the offspring of enriched mutant parents. The transgenerational transmission of this effect occurs from the enriched mother to her offspring during embryogenesis.

Perspective: If a similar phenomenon occurs in humans, the effectiveness of one's memory during adolescence, particularly in those with defective cell signaling mechanisms that control memory, can be influenced by environmental stimulation experienced by one's mother during her youth.

An enriched environment during young age causes striking changes in brain function including enhanced learning and memory. This group showed previously that 2 weeks of enriched environment induces the expression of a signaling cascade that contributes to the induction of synaptic plasticity that is known to be important for learning and memory - the appearance of an otherwise latent NMDA receptor/cAMP/p38 MAP kinase signaling pathway. They now demonstrate the incredible power of an animal's environment to modulate this signaling network and to improve contextual fear memory formation across generations. Exposing young animals to an enriched environment enhanced long-term potentiation induction in their future offspring through adolescence, even if the offspring are not exposed to enriched environment. In both generations, this phenomenon involves the addition of CAMP and p38 MAP kinase dependence to LTP induction. If this reminds you of transgenerational transmission of epigenetic changes, such changes have not been investigated here. It was previously shown that pups, who are exposed to strong nurturing mothers, display lifelong alterations in the patterns of promoter DNA methylation and changes in expression of specific genes in the hypothalamus that regulate the stress response. These offspring then become strong nurturing mothers and pass the phenotype on to the next generation by their behavior. 


\section{DNA methylation profiles in monozygotic and dizygotic twins}

Kaminsky ZA, Tang T, Wang SC, Ptak C, Oh GH, Wong AH, Feldcamp LA, Virtanen C, Halfvarson J, Tysk C, McRae AF, Visscher PM, Montgomery GW, Gottesman, II, Martin NG, Petronis A

Centre for Addiction and Mental Health, Toronto, Ont., Canada

Nat Genet 2009;41:240-245

Context: Twin studies have provided the basis for genetic and epidemiological studies in human complex traits.

Methods: As epigenetic factors can contribute to phenotypic outcomes, the authors conducted a DNA methylation analysis in white blood cells (WBC), buccal epithelial cells and gut biopsies of 114 monozygotic (MZ) twins as well as WBC and buccal epithelial cells of 80 dizygotic (DZ) twins using 12K CpG island microarrays.

Results: They provide the first annotation of epigenetic metastability of 6,000 unique genomic regions in $\mathrm{MZ}$ twins. An intraclass correlation (ICC)-based comparison of matched MZ and DZ twins showed significantly higher epigenetic difference in buccal cells of DZ co-twins ( $p=1.210-294)$.

Conclusions: Although such higher epigenetic discordance in DZ twins can result from DNA sequence differences, these in silico SNP analyses and animal studies favor the hypothesis that it is due to epigenomic differences in the zygotes, suggesting that molecular mechanisms of heritability may not be limited to DNA sequence differences.

MZ twins reared apart are generally quite similar to $M Z$ twins reared together according to IQ, personality, social attitudes and more. Yet variations are not rare. These investigators mapped MZ twin DNA methylation differences in relatively easily accessible tissues: white blood cells, buccal epithelial cells and rectal biopsies. They detected a large degree of co-twin DNA methylation variation in all tissues investigated. The varying degrees of epigenetic dissimilarity detected between MZ twins may reflect differences in epigenetic divergence among embryonic cells at the time the twin blastomeres separated. They found greater epigenetic similarity between $\mathrm{MZ}$ co-twins at functionally important regions in comparison to the loci without clearly defined regulatory function, suggesting functional stratification of the epigenome. They speculate that stochastic events in epigenetically determined phenotypic differences in MZ co-twins are much more important than environment.

\section{Super persistent predator}

\section{Human predators outpace other agents of trait change in the wild}

Darimont CT, Carlson SM, Kinnison MT, Paquet PC, Reimchen TE, Wilmers CC Department of Biology, University of Victoria, Victoria, B.C., Canada

darimont@ucsc.edu

Proc Natl Acad Sci USA 2009;106:952-954

Context: The observable traits of wild populations are continually shaped and reshaped by the environment and numerous agents of natural selection, including predators. In stark contrast with most predators, humans now typically exploit high proportions of prey populations and target large, reproductive-aged adults. Consequently, organisms subject to consistent and strong 'harvest selection' by fishers, hunters, and plant harvesters may be expected to show particularly rapid and dramatic changes in phenotype. However, a comparison of the rate at which phenotypic changes in exploited taxa occurs relative to other systems has never been undertaken.

Results: Average phenotypic changes in 40 human-harvested systems are much more rapid than changes reported in studies examining not only natural $(\mathrm{n}=20$ systems) but also other human-driven $(\mathrm{n}=25$ systems) perturbations in the wild, outpacing them by $>300$ and $50 \%$, respectively. Accordingly, harvested organisms show some of the most abrupt trait changes ever observed in wild populations, pro- 
viding a new appreciation for how fast phenotypes are capable of changing. These changes, which include average declines of almost $20 \%$ in size-related traits and shifts in life history traits of nearly $25 \%$, are most rapid in commercially exploited systems and, thus, have profound conservation and economic implications.

Conclusions: The widespread potential for transitively rapid and large effects on size- or life history-mediated ecological dynamics might imperil populations, industries, and ecosystems.

Previous research has shown that in response to commercial fishing and hunting, traits such as average size at reproductive age adjust. Human prey mammals and fish turned smaller by $20 \%$ and reproduced earlier, which made them even more vulnerable. From a meta-analysis of reports on the morphology and life histories of 29 species, including fish, mammals and plants, these authors report that phenotypic changes in human-harvested organisms occurred more than $300 \%$ faster than in natural systems, and $50 \%$ faster than in systems affected by other human influences, such as pollution. Predation by humans drives phenotypic changes in exploited prey much faster than other evolutionary pressures do. Prey animals adjust their life history to a new, shorter lifespan. Why does human predation work so quickly? Apparently, because it is often felt by a large proportion of the adults in a population. Why is human prey different from wild animals prey? Whereas the latter hunt the small and weak, humans' hunting and fishing techniques and targets are the big and strong. This is paradoxically aggravated by regulations that forbid the hunting or fishing of small animals.

\section{Artificial organism}

\section{One-step assembly in yeast of 25 overlapping DNA fragments to form a complete synthetic Mycoplasma genitalium genome}

Gibson DG, Benders GA, Axelrod KC, Zaveri J, Algire MA, Moodie M, Montague MG, Venter JC, Smith HO, Hutchison CA, 3rd

The J. Craig Venter Institute, Synthetic Biology Group, Rockville, Md., USA

dgibson@jcvi.org

Proc Natl Acad Sci USA 2008;105:20404-20409

Context: This group previously reported assembly and cloning of the synthetic Mycoplasma genitalium JCVI-1.0 genome in the yeast Saccharomyces cerevisiae by recombination of six overlapping DNA fragments to produce a $592-\mathrm{kb}$ circle.

Results: Here they extend this approach by demonstrating assembly of the synthetic genome from 25 overlapping fragments in a single step.

Conclusions: The use of yeast recombination greatly simplifies the assembly of large DNA molecules from both synthetic and natural fragments.

The Yearbook 2008 missed this breakthrough, published in February 2008 [Gibson DG, et al: Science 2008;319(5867):1215-1220], but this latest in the series cannot go unmentioned. By stitching together more than 500,000 bp of DNA, they created the first synthetic genome as a step towards the construction of the world's first artificial organism. 


\section{Speciation through sensory drive in cichlid fish}

Seehausen O, Terai Y, Magalhaes IS, Carleton KL, Mrosso HD, Miyagi R, van der Sluijs I, Schneider MV, Maan ME, Tachida H, Imai H, Okada N

Institute of Zoology, University of Bern, Bern, Switzerland

ole.seehausen@aqua.unibe.ch

Nature 2008;455:620-626

Context: Theoretically, divergent selection on sensory systems can cause speciation through sensory drive. However, empirical evidence is rare and incomplete.

Results: This study demonstrates sensory drive speciation within island populations of cichlid fish. They identify the ecological and molecular basis of divergent evolution in the cichlid visual system, demonstrate associated divergence in male coloration and female preferences, and show subsequent differentiation at neutral loci, indicating reproductive isolation. Evidence is replicated in several pairs of sympatric populations and species. Variation in the slope of the environmental gradients explains variation in the progress towards speciation: speciation occurs on all but the steepest gradients.

Conclusions: This is the most complete demonstration so far of speciation through sensory drive without geographical isolation. The results also provide a mechanistic explanation for the collapse of cichlid fish species diversity during the anthropogenic eutrophication of Lake Victoria.

Classically, geographical isolation was considered to be the main drive for speciation. We now learn of a sensory drive that leads to the evolution of color polymorphisms and speciation, even in the absence of geographical isolation, when the light environment is heterogeneous. The cichlids of the African lakes are the most rapidly speciating species known. Males are either red or blue, and females have genetic variation for visual sensitivity to those colors. Females with blue-biased vision seem to mate only with blue males, whereas red-biased females mate only with red males. The implication is that natural selection acting on the visual system contributes to the formation of new species.

\section{Of drink and love}

\section{Genetic variation in the vasopressin receptor 1a gene (AVPR1A) associates with pair-bonding behavior in humans}

Walum H, Westberg L, Henningsson S, Neiderhiser JM, Reiss D, Igl W, Ganiban JM, Spotts EL, Pedersen NL, Eriksson

E, Lichtenstein $\mathrm{P}$

Department of Medical Epidemiology and Biostatistics, Karolinska Institutet, Stockholm, Sweden

hasse.walum@ki.se

Proc Natl Acad Sci USA 2008;105:14153-14156

Context: Pair-bonding has been suggested to be a critical factor in the evolutionary development of the social brain. The brain neuropeptide arginine vasopressin (AVP) exerts an important influence on pairbonding behavior in voles. There is a strong association between a polymorphic repeat sequence in the 5 '-flanking region of the gene (AVPR1A) encoding one of the AVP receptor subtypes (V1aR), and proneness for monogamous behavior in males of this species. It is not yet known whether similar mechanisms are important also for human pair-bonding.

Results: This report shows an association between one of the human AVPR1A repeat polymorphisms (RS3) and traits reflecting pair-bonding behavior in men, including partner bonding, perceived marital problems, and marital status. It shows that the RS3 genotype of the males also affects marital quality as perceived by their spouses.

Conclusions: These results suggest an association between a single gene and pair-bonding behavior in humans, and indicate that the well-characterized influence of AVP on pair-bonding in voles may be of relevance also for humans. 
The topic here is pair-bonded relationships, or what we call love. It may have been the demands for pair-bonding behavior that triggered the evolutionary development of the primate social brain. It has known for some time that oxytocin is involved in mother-infant bonding, but also between man and woman. We now learn that AVP, acting through the pressor receptor V1aR, plays a role in the regulation of love behaviors. Polymorphisms of the receptor gene are associated with variation in relation quality; men with a certain repeat polymorphism were twice as likely to remain unmarried, or when married, twice as likely to report marriage crises. You can already purchase on the internet a love-boosting oxytocin and pheromones preparation. AVP may make this love mixture more effective. You may wish to study the bonding of your patients with diabetes insipidus.

\title{
A second hit
}

\section{Persistent hypomethylation in the promoter of nucleosomal binding protein 1 (Nsbp1) correlates with overexpression of Nsbp1 in mouse uteri neonatally exposed to diethylstilbestrol or genistein}

\author{
Tang WY, Newbold R, Mardilovich K, Jefferson W, Cheng RY, Medvedovic M, Ho SM \\ Department of Environmental Health, Kettering Complex, University of Cincinnati Medical Center, Cincinnati, Ohio, \\ USA \\ Endocrinology 2008;149:5922-5931
}

Context: Neonatal exposure of CD-1 mice to diethylstilbestrol (DES) or genistein (GEN) induces uterine adenocarcinoma in aging animals. Uterine carcinogenesis in this model is ovarian dependent because its evolution is blocked by prepubertal ovariectomy.

Aim: This study seeks to discover novel uterine genes whose expression is altered by such early endocrine disruption via an epigenetic mechanism.

Methods: Neonatal mice were treated with 1 or $1,000 \mu \mathrm{g} / \mathrm{kg}$ DES, $50 \mathrm{mg} / \mathrm{kg} \mathrm{GEN}$, or oil (control) on days 1-5. One group of treated mice was killed before puberty on day 19. Others were ovariectomized or left intact, and killed at 6 and 18 months of age. Methylation-sensitive restriction fingerprinting was performed to identify differentially methylated sequences associated with neonatal exposure to DES/GEN. Results: Among 14 candidates, nucleosomal binding protein 1 (Nsbp1), the gene for a nucleosome-coreparticle binding protein, was selected for further study because of its central role in chromatin remodeling. In uteri of immature control mice, Nsbp1 promoter CpG island (CGI) was minimally methylated. Once control mice reached puberty, the Nsbp1 CGI became hypermethylated, and gene expression declined further. In contrast, in neonatal DES/GEN-treated mice, the Nsbp1 CGI stayed anomalously hypomethylated, and the gene exhibited persistent overexpression throughout life. However, if neonatal DES/GEN-treated mice were ovariectomized before puberty, the CGI remained minimally to moderately methylated, and gene expression was subdued except in the group treated with $1,000 \mu \mathrm{g} / \mathrm{kg}$ DES. Conclusions: The life reprogramming of uterine Nsbp 1 expression by neonatal DES/GEN exposure appears to be mediated by an epigenetic mechanism that interacts with ovarian hormones in adulthood.

Exposure to estrogenic chemicals during critical stages of development exerts long-lasting effects on male and female reproductive organs. The best-studied example is exposure to diethylstilbestrol (DES) and the risk for clear cell adenocarcinoma of the vagina and cervix in female offspring (DES daughters). In contrast to in utero exposure to xenoestrogens, peripubertal human exposures to dietary phytoestrogens such as genistein (GEN) appear to reduce later-life breast cancer risk. Yet, questions related to the safety of early-life exposure to phytoestrogens remain unanswered. Infants exposed to high levels of dietary phytoestrogens have an increased risk of developing type 1 diabetes. This study aimed to determine whether neonatal exposure to DES/GEN can alter gene expression in the mouse uterus in later life via an epigenetic mechanism such as DNA methylation, and looked for interaction between adult ovarian hormones and neonatal DES/GEN reprogramming. The study identified 14 candidate genes that encode proteins involved in signal transduction, receptor activation, tumor angiogenesis/metastasis, cell proliferation, apoptosis, intracellular trafficking, DNA repair, and chromatin remodeling, whose methylation status in the mature mouse uterus was altered by 
neonatal exposure of the pups to DES and/or GEN. The discovery of these genes has strengthened the claim of an epigenetic basis for the developmental effects of DES, and has provided much needed initial evidence that a phytoestrogen such as GEN may possess a similar potential. The complex interplay among the type of estrogen, timing of exposure, reproductive status, and aging time line all significantly contribute to the phenotypical outcome of the epigenetic reprogramming in this model system.

The main novelty of this article is in the demonstration that epigenetic reprogramming requires a 'second hit'. The onset of uterine adenocarcinoma in neonatal DES-exposed mice required a secondary hormonal 'push' by pubertal steroids because prepubertally ovariectomized mice did not develop cancers. This is a direct function of specific DNA methylation changes induced by the secondary pubertal hormones that occur only in mice that were first exposed neonatally to DES or GEN.

\section{Reduced by fat}

\section{7-0xysterols modulate glucocorticoid activity in adipocytes through competition for 11ß-hydroxysteroid dehydrogenase type 1}

Wamil M, Andrew R, Chapman KE, Street J, Morton NM, Seckl JR

Endocrinology Unit, Centre for Cardiovascular Science, The Queen's Medical Research Institute, University of Edinburgh, Edinburgh, UK

Endocrinology 2008;149:5909-5918

Context: Obesity is associated with an increased risk of diabetes type 2, dyslipidemia, and atherosclerosis. These cardiovascular and metabolic abnormalities are exacerbated by excessive dietary fat, particularly cholesterol and its metabolites. High adipose tissue glucocorticoid levels, generated by the intracellular enzyme $11 \beta$-hydroxysteroid dehydrogenase type $1(11 \beta-H S D 1)$, are also implicated in the pathogenesis of obesity, metabolic syndrome, and atherosclerosis. $11 \beta-\mathrm{HSD} 1$ also interconverts the atherogenic oxysterols 7 -ketocholesterol $(7 \mathrm{KC})$ and $7 \beta$-hydroxycholesterol $(7 \beta-\mathrm{HC})$.

Aim: Here, they report that $11 \beta-\mathrm{HSD} 1$ catalyzes the reduction of $7 \mathrm{KC}$ to $7 \beta-\mathrm{HC}$ in mature $3 \mathrm{~T} 3-\mathrm{L} 1$ and 3T3-F442A adipocytes, leading to cellular accumulation of 7 $\beta-\mathrm{HC}$.

Results: Approximately $73 \%$ of added $7 \mathrm{KC}$ was reduced to $7 \beta-\mathrm{HC}$ within $24 \mathrm{~h}$; this conversion was prevented by selective inhibition of $11 \beta-H S D 1$. Oxysterol and glucocorticoid conversion by $11 \beta-H S D 1$ was competitive and occurred with a physiologically relevant $\mathrm{IC}_{50}$ range of $450 \mathrm{~nm}$ for $7 \mathrm{KC}$ inhibition of glucocorticoid metabolism. Working as an inhibitor of $11 \beta$-reductase activity, $7 \mathrm{KC}$ decreased the regeneration of active glucocorticoid and limited the process of differentiation of 3T3-L1 preadipocytes. $7 \mathrm{KC}$ and $7 \beta-\mathrm{HC}$ did not activate liver $\mathrm{X}$ receptor in a transactivation assay, nor did they display intrinsic activation of the glucocorticoid receptor. However, when coincubated with glucocorticoid (10 $\mathrm{nm}), 7 \mathrm{KC}$ repressed, and $7 \beta-\mathrm{HC}$ enhanced glucocorticoid receptor transcriptional activity. The effect of 7 -oxysterols resulted from the modulation of $11 \beta$-HSD1 reaction direction, and could be ameliorated by overexpression of hexose 6-phosphate dehydrogenase, which supplies reduced nicotinamide adenine dinucleotide phosphate to $11 \beta-H S D 1$.

Conclusions: The activity and reaction direction of adipose $11 \beta-H S D 1$ is altered under conditions of oxysterol excess, and could impact upon the pathophysiology of obesity and its complications.

The reduction of inert cortisone to active cortisol by $11 \beta$-HSD1, which is elevated specifically in adipose tissue of obese humans and rodents, was suggested to cause 'Cushing's syndrome of adipose tissue' in idiopathic obesity. Transgenic mice overexpressing 11 $\beta$-HSD1 selectively in adipose tissue develop many features of the metabolic syndrome, including glucose intolerance, insulin resistance, dyslipidemia, and hypertension, whereas $11 \beta$-HSD1 null mice are protected from these deleterious effects upon high-fat feeding and have an atheroprotective phenotype, with increased plasma HDL cholesterol and lower free fatty acid levels. It was reported recently that $11 \beta$-HSD1 interconverts in the liver 7-ketocholesterol $(7 \mathrm{KC})$ and $7 \beta$-hydroxycholesterol $(7 \beta-\mathrm{HC})$, which are the most abundant oxysterols in oxLDL, and their concentrations correlate with atherosclerosis risk. Given the key role of adipose tissue in modulating whole-body cholesterol homeostasis, the study hypothesized that 
metabolism of these oxysterols by $11 \beta$-HSD 1 might provide a novel link between obesity, adipose glucocorticoid action, and atherogenesis. The findings show that 7-oxysterols are produced enzymatically in adipocytes, regulate 11ß-HSD1-dependent conversion of glucocorticoids, and, therefore, signaling by glucocorticoid receptor, and when in excess may inhibit the differentiation of preadipocytes. The effects appear to occur predominantly through substrate competition at the enzyme activity level, and may also include reversal of $11 \beta-H S D 1$ reaction direction (reductase) if $7 \mathrm{KC}$ levels are high and reduced nicotinamide adenine dinucleotide phosphate cofactor levels are limiting within the cell. These data could lead to a revision of our understanding of the association between glucocorticoids and atherogenesis.

Whereas essential for preadipocyte differentiation, in the mature adipocytes glucocorticoids promote lipolysis, increasing glycerol release that can lead to insulin resistance under states of pathogenic excess. The data presented here further define the mechanisms and components underlying this biology in which increased levels of oxysterols, such as that found in dyslipidemia and obesity, can modulate the enzymatic activity controlling glucocorticoid production.

\section{A pluripotent future: three papers on stem cells}

\section{Induction of pluripotent stem cells from primary human fibroblasts with only Oct4 and Sox2}

Huangfu D, Osafune K, Maehr R, Guo W, Eijkelenboom A, Chen S, Muhlestein W, Melton DA

Department of Stem Cell and Regenerative Biology, Howard Hughes Medical Institute, Harvard Stem Cell Institute, Harvard University, Cambridge, Mass., USA

Nat Biotechnol 2008;26:1269-1275

Context: Ectopic expression of defined sets of genetic factors can reprogram somatic cells to induced pluripotent stem (iPS) cells that closely resemble embryonic stem (ES) cells. The low efficiency with which iPS cells are derived hinders studies on the molecular mechanism of reprogramming, and integration of viral transgenes, in particular the oncogenes c-Myc and Klf4, may handicap this method for human therapeutic applications.

Results: Valproic acid (VPA), a histone deacetylase inhibitor, enables reprogramming of primary human fibroblasts with only two factors, Oct4 and Sox2, without the need for the oncogenes c-Myc or Klf4. The two factor-induced human iPS cells resemble human ES cells in pluripotency, global gene expression profiles and epigenetic states.

Conclusions: These results support the possibility of reprogramming through purely chemical means, which would make therapeutic use of reprogrammed cells safer and more practical.

\section{Induced pluripotent stem cells generated without viral integration}

Stadtfeld M, Nagaya M, Utikal J, Weir G, Hochedlinger K

Massachusetts General Hospital Cancer Center and Center for Regenerative Medicine, Boston, Mass., USA

Science 2008;322:945-949

Pluripotent stem cells have been generated from mouse and human somatic cells by viral expression of the transcription factors Oct4, Sox2, Klf4, and c-Myc. A major limitation of this technology is the use of potentially harmful genome-integrating viruses. We generated mouse-induced pluripotent stem (iPS) cells from fibroblasts and liver cells by using nonintegrating adenoviruses transiently expressing Oct4, Sox2, Klf4, and c-Myc. These adenoviral iPS (adeno-iPS) cells show DNA demethylation characteristic of reprogrammed cells, express endogenous pluripotency genes, form teratomas, and contribute to multiple tissues, including the germ line, in chimeric mice. Our results provide strong evidence that insertional mutagenesis is not required for in vitro reprogramming. Adenoviral reprogramming may provide an improved method for generating and studying patient-specific stem cells and for comparing embryonic stem cells and iPS cells. 


\section{Disease-specific induced pluripotent stem cells}

Park IH, Arora N, Huo H, Maherali N, Ahfeldt T, Shimamura A, Lensch MW, Cowan C, Hochedlinger K, Daley GQ Department of Medicine, Division of Pediatric Hematology Oncology, Children's Hospital Boston, and Dana-Farber Cancer Institute, Boston, Mass., USA

Cell 2008;134:877-886

Context: Tissue culture of immortal cell strains from diseased patients is an invaluable resource for medical research but is largely limited to tumor cell lines or transformed derivatives of native tissues.

Aim: The authors describe the generation of induced pluripotent stem (iPS) cells from patients with a variety of genetic diseases with either mendelian or complex inheritance; these diseases include adenosine deaminase deficiency-related severe combined immunodeficiency (ADA-SCID), Shwachman-BodianDiamond syndrome (SBDS), Gaucher disease (GD) type III, Duchenne (DMD) and Becker muscular dystrophy (BMD), Parkinson disease (PD), Huntington disease (HD), juvenile-onset, type 1 diabetes mellitus (JDM), Down syndrome (DS)/trisomy 21, and the carrier state of Lesch-Nyhan syndrome.

Conclusions: Such disease-specific stem cells offer an unprecedented opportunity to recapitulate both normal and pathologic human tissue formation in vitro, thereby enabling disease investigation and drug development.

Following the reprogramming of human skin cells to imitate embryonic stem cells, researches have now used adult skin cells and by adding valproic acid they reverted the cells back into a stem cell-like state. Scientists were also able to reprogram adult cells into pluripotent stem cells without utilization of retroviruses. Stem cell researchers believe that patient-derived stem cells will be needed to develop into different tissue types, hoping to identify the biological errors that contribute to these diseases. This is now achieved for disorders such as Parkinson's disease and Down's syndrome.

\section{Correcting for purifying selection: an improved human mitochondrial molecular clock}

Soares P, Ermini L, Thomson N, Mormina M, Rito T, Rohl A, Salas A, Oppenheimer S, Macaulay V, Richards MB Institute of Integrative and Comparative Biology, Faculty of Biological Sciences, University of Leeds, Leeds, UK Am J Hum Genet 2009;84:740-759

Context: There is currently no calibration available for the whole human mtDNA genome, incorporating both coding and control regions. Furthermore, as several authors have pointed out recently, linear molecular clocks that incorporate selectable characters are in any case problematic.

Objective: This paper confirms a modest effect of purifying selection on the mtDNA coding region and proposes an improved molecular clock for dating human mtDNA, based on a worldwide phylogeny of $>2,000$ complete mtDNA genomes and calibrating against recent evidence for the divergence time of humans and chimpanzees.

Results: They focus on a time-dependent mutation rate based on the entire mtDNA genome and supported by a neutral clock based on synonymous mutations alone. They show that the corrected rate is further corroborated by archaeological dating for the settlement of the Canary Islands and Remote Oceania and also, given certain phylogeographic assumptions, by the timing of the first modern human settlement of Europe and resettlement after the Last Glacial Maximum. The corrected rate yields an age of modern human expansion in the Americas at approximately 15 thousand years that - unlike the uncorrected clock - matches the archaeological evidence, but continues to indicate an out-of-Africa dispersal at around 55-70 thousand years, 5-20 thousand years before any clear archaeological record, suggesting the need for archaeological research efforts focusing on this time window. They also present improved rates for the mtDNA control region, and the first comprehensive estimates of positional mutation rates for human mtDNA, which are essential for defining mutation models in phylogenetic analyses.

These researchers have devised a method of dating ancient human migration based on mitochondrial DNA (mtDNA) calculation by taking into account the process of natural selection, which very gradually but nonlinearly removes harmful gene mutations in the mtDNA. This produces a timedependent effect on how many mutations you see in the family tree, to provide reliable time-depth estimates for the entire window of anatomically modern human evolution. They can now put a timescale on any part of a given family tree, right back to 'Mitochondrial Eve', who lived some 200,000 
years ago. Working with published databases of more than 2,000 fully sequenced mtDNAs, the new method yielded some surprising findings. They confirm the date mankind's expansion through the Americas to be some 15,000 years ago, around the time of the first unequivocal archaeological remains, and estimate the time of the third 'out of Africa' migration around $60-70,000$ years ago some 10-20,000 years earlier than previously thought. The paper offers a calculator into which researchers can feed their data.

\title{
Concepts revised: screening for disease is not a panacea
}

\author{
Screening and prostate-cancer mortality in a randomized European study \\ Schroder FH, Hugosson J, Roobol MJ, Tammela TL, Ciatto S, Nelen V, Kwiatkowski M, Lujan M, Lilja H, Zappa M, \\ Denis LJ, Recker F, Berenguer A, Maattanen L, Bangma CH, Aus G, Villers A, Rebillard X, van der Kwast T, Blijenberg \\ BG, Moss SM, de Koning HJ, Auvinen A \\ Department of Urology, Erasmus Medical Center, Rotterdam, The Netherlands
}

N Engl J Med 2009;360:1320-1328

Background: The European Randomized Study of Screening for Prostate Cancer was initiated in the early 1990s to evaluate the effect of screening with prostate-specific-antigen (PSA) testing on death rates from prostate cancer.

Methods: 182,000 men between the ages of 50 and 74 years through registries in seven European countries were identified for inclusion. The men were randomly assigned to a group that was offered PSA screening at an average of once every 4 years or to a control group that did not receive such screening. The predefined core age group for this study included 162,243 men between the ages of 55 and 69 years. The primary outcome was the rate of death from prostate cancer. Mortality follow-up was identical for the two study groups and ended on December 31, 2006.

Results: In the screening group, $82 \%$ of men accepted at least one offer of screening. During a median follow-up of 9 years, the cumulative incidence of prostate cancer was $8.2 \%$ in the screening group and $4.8 \%$ in the control group. The rate ratio for death from prostate cancer in the screening group, as compared with the control group, was 0.80 (95\% confidence interval $[\mathrm{CI}], 0.65-0.98$; adjusted $\mathrm{p}=$ 0.04). The absolute risk difference was 0.71 deaths per 1,000 men. This means that 1,410 men would need to be screened and 48 additional cases of prostate cancer would need to be treated to prevent 1 death from prostate cancer. The analysis of men who were actually screened during the first round (excluding subjects with noncompliance) provided a rate ratio for death from prostate cancer of 0.73 (95\% CI, 0.56-0.90).

Conclusions: PSA-based screening reduced the rate of death from prostate cancer by $20 \%$ but was associated with a high risk of overdiagnosis.

\section{Mortality results from a randomized prostate-cancer screening trial}

Andriole GL, Crawford ED, Grubb RL, 3rd, Buys SS, Chia D, Church TR, Fouad MN, Gelmann EP, Kvale PA, Reding DJ, Weissfeld JL, Yokochi LA, O'Brien B, Clapp JD, Rathmell JM, Riley TL, Hayes RB, Kramer BS, Izmirlian G, Miller AB, Pinsky PF, Prorok PC, Gohagan JK, Berg CD

Washington University School of Medicine, St. Louis, Mo.,USA

N Engl J Med 2009;360:1310-1319

Background: The effect of screening with prostate-specific-antigen (PSA) testing and digital rectal examination (DRE) on the rate of death from prostate cancer is unknown. This is the first report from the Prostate, Lung, Colorectal, and Ovarian (PLCO) Cancer Screening Trial on prostate-cancer mortality. Methods: From 1993 through 2001, 76,693 men at 10 US study centers were randomly assigned to receive either annual screening (38,343 subjects) or usual care as the control (38,350 subjects). Men in the screening group were offered annual PSA testing for 6 years and DRE for 4 years. The subjects and healthcare providers received the results and decided on the type of follow-up evaluation. Usual care sometimes included screening, as some organizations have recommended. The numbers of all cancers and deaths and causes of death were ascertained. 
Results: In the screening group, rates of compliance were $85 \%$ for PSA testing and $86 \%$ for DRE. Rates of screening in the control group increased from $40 \%$ in the first year to $52 \%$ in the sixth year for PSA testing and ranged from 41 to $46 \%$ for DRE. After 7 years of follow-up, the incidence of prostate cancer per 10,000 person-years was 116 (2,820 cancers) in the screening group and 95 (2,322 cancers) in the control group (rate ratio, 1.22; 95\% confidence interval [CI], 1.16-1.29). The incidence of death per 10,000 person-years was 2.0 (50 deaths) in the screening group and 1.7 (44 deaths) in the control group (rate ratio, $1.13 ; 95 \% \mathrm{CI}, 0.75-1.70$ ). The data at 10 years were $67 \%$ complete and consistent with these overall findings.

Conclusions: After 7-10 years of follow-up, the rate of death from prostate cancer was very low and did not differ significantly between the two study groups.

\section{Long-term health and quality-of-life consequences of mass screening for childhood celiac disease: a 10-year follow-up study}

Van Koppen EJ, Schweizer JJ, Csizmadia CG, Krom Y, Hylkema HB, van Geel AM, Koopman HM, Verloove-Vanhorick $\mathrm{SP}$, Mearin $\mathrm{ML}$

Department of Paediatrics, Leiden University Medical Centre, Leiden, The Netherlands

Pediatrics 2009;123:e582-588

Objective: Mass screening for celiac disease is controversial. The objective of the study was to determine whether detection of childhood celiac disease by mass screening improves long-term health status and health-related quality of life.

Methods: A prospective 10-year follow-up study of 32 children who were aged 2-4 years, had celiac disease identified by mass screening, and had a gluten-free diet (19) or a normal gluten-containing diet (13) was conducted. The follow-up included assessments of general health status, celiac disease-associated symptoms, celiac disease-associated serum antibodies, and health-related quality of life.

Results: Ten years after mass screening, $81 \%$ of the children were adhering to a gluten-free diet. The health status improved in $66 \%$ of the treated children: in $41 \%$ by early treatment and in $25 \%$ by prevention of the gluten-dependent symptoms that they developed after diagnosis. For $19 \%$ of the children, treatment after screening would not have improved their health status, because they had no symptoms at screening and have remained symptom-free while consuming gluten. The health-related quality of life of the children with symptoms improved significantly after 1 year of gluten-free diet. Ten years after screening, the health-related quality of life of the children with celiac disease was similar to that of the reference population.

Conclusion: Identification by mass screening led 10 years later to health improvement in $66 \%$ of children without deterioration of generic health-related quality of life. There is a good compliance after mass screening. In a research setting, delaying treatment for children without symptoms seems to be an option after a positive screening test. Long-term follow-up studies are needed to assess possible longterm complications in untreated, nonsymptomatic celiac disease.

Including papers on prostate cancer screening in the Science and Medicine section of the Yearbook does not reflect a personal concern but rather the quality of these two papers that exemplify how an obviously good idea might in the end prove not to be so good. These two papers used screening strategies for prostate cancer in more than 200,000 subjects, based on PSA measurements, with or without rectal examination. There was a $20 \%$ reduction by screening of death related to prostate cancer in the European study and no difference in the US study. In all cases, the number of cancer cases detected was higher with screening (twice as many in the European study). In the positive European study, the estimated absolute reduction in cancer-related mortality of about 0.7 deaths per 1,000 men should be weighed against the interventions. The 73,000 men in the screening group had more than 17,000 biopsies. Diagnosis led to more treatment: 277 vs. 100 per 10,000 radical prostatectomy and 220 vs. 123 per 10,000 radiation therapy with or without hormones. Altogether, these huge studies should be praised, although specialists do not consider them as definitive [1].

The study by the Dutch group is obviously more modest (32 celiac children identified by mass screening) but asks the important question of whether the screening process has improved long-term quality of life, rather than focusing on the ability of the screening to identify the disease. The authors conclude that in $66 \%$ of the children with a diagnosis made after screening, the health status improved. However, they fail to take into account the roughly equal number of children who had a small bowel 
biopsy with possible impact on discomfort of an unnecessary procedure. Altogether, these studies emphasize the need for evidence: nothing should be taken for granted in the screening world. Hard and meaningful endpoints should be used to evaluate all our medical actions in that domain.

\section{New mechanisms: fasting against cancer}

\section{Tumours with PI3K activation are resistant to dietary restriction}

Kalaany NY, Sabatini DM

Whitehead Institute for Biomedical Research, Nine Cambridge Center, Cambridge, Mass., USA

Nature 2009;458:725-731

Background: Dietary restriction delays the incidence and decreases the growth of various types of tumors, but the mechanisms underlying the sensitivity of tumors to food restriction remain unknown.

Results: Certain human cancer cell lines, when grown as tumor xenografts in mice, are highly sensitive to the anti-growth effects of dietary restriction, whereas others are resistant. Cancer cells that form dietaryrestriction-resistant tumors carry mutations that cause constitutive activation of the phosphatidylinositol-3-kinase (PI3K) pathway and in culture proliferate in the absence of insulin or insulin-like growth factor 1. Substitution of an activated mutant allele of PI3K with wild-type PI3K in otherwise isogenic cancer cells, or the restoration of PTEN expression in a PTEN-null cancer cell line, is sufficient to convert a dietary-restriction-resistant tumour into one that is dietary-restriction-sensitive. Dietary restriction does not affect a PTEN-null mouse model of prostate cancer, but it significantly decreases tumour burden in a mouse model of lung cancer lacking constitutive PI3K signalling.

Conclusions: The PI3K pathway is an important determinant of the sensitivity of tumors to dietary restriction, and activating mutations in the pathway may influence the response of cancers to dietary restriction-mimetic therapies.

Dietary restriction limiting nutrient intake without reaching malnutrition extends lifespan in most if not all, species including humans [2]. This extension of lifespan is partly due to a decreased rate of cancer, and the growth of certain cancers can be limited by dietary restriction. This surprising benefit is likely to be due to the evolutionary advantage of prolonging lifespan under suboptimal nutrient availability, and postponing reproduction until food is abundant. The authors identified human cancer lines that when implanted in mice were sensitive or insensitive to the effect of dietary restriction and looked for the mechanism underlying this differential effect. They found that PI3 kinase, enzyme acting downstream of the insulin and the IGF-1 receptor is involved in this differential effect through a mechanism of apoptosis, and that constitutive activation of the PI3K pathway abolishes the effect of dietary restriction. Another component of the pathway, PTEN, is also similarly involved. These observations link once again the insulin and IGF-1 pathway to longevity and cancer [3]. Indeed, mutation of the GH receptor, which affects the production of IGF1, abrogates the dietary restrictioninduced longevity in mice. These mechanisms may be related to the recent worrisome news of the potentially increased risk of cancer with the use of glargine insulin, together with the decreased risk associated with the use of metformin [4].

\section{Pulmonary autoimmunity as a feature of autoimmune polyendocrine syndrome type 1 and identification of KCNRG as a bronchial autoantigen}

Alimohammadi M, Dubois N, Skoldberg F, Hallgren A, Tardivel I, Hedstrand H, Haavik J, Husebye ES, Gustafsson J, Rorsman F, Meloni A, Janson C, Vialettes B, Kajosaari M, Egner W, Sargur R, Ponten F, Amoura Z, Grimfeld A, De Luca F, Betterle C, Perheentupa J, Kampe O, Carel JC

Department of Medical Sciences, University Hospital, Uppsala University, Uppsala, Sweden

mohammad.alimohammadi@medsci.uu.se

Proc Natl Acad Sci USA 2009;106:4396-4401

Background: Patients with autoimmune polyendocrine syndrome type 1 (APS-1) suffer from multiple organ-specific autoimmunity with autoantibodies against target tissue-specific autoantigens. Endocrine 
and nonendocrine organs such as skin, hair follicles, and liver are targeted by the immune system. Despite sporadic observations of pulmonary symptoms among APS-1 patients, an autoimmune mechanism for pulmonary involvement has not been elucidated.

Methods: A subset of APS-1 patients with respiratory symptoms is reported. Eight patients with pulmonary involvement were identified. Severe airway obstruction was found in 4 patients, leading to death in 2. Immunoscreening of a cDNA library using serum samples from a patient with APS-1 and obstructive respiratory symptoms identified a putative potassium channel regulator (KCNRG) as a pulmonary autoantigen. Reactivity to recombinant KCNRG was assessed in 110 APS-1 patients by using immunoprecipitation.

Results: Autoantibodies to KCNRG were present in 7 of the 8 patients with respiratory symptoms, but in only 1 of 102 APS-1 patients without respiratory symptoms. Expression of KCNRG messenger RNA and protein was found to be predominantly restricted to the epithelial cells of terminal bronchioles.

Conclusions: Autoantibodies to KCNRG, a protein mainly expressed in bronchial epithelium, are strongly associated with pulmonary involvement in APS-1. These findings may facilitate the recognition, diagnosis, characterization, and understanding of the pulmonary manifestations of APS- 1 .

Sorry for the lack of modesty in citing an own contribution. Starting from a single observation of an unusual component of a very rare disease, a new concept of autoimmune bronchiolitis could be proposed, and that a molecular autoimmune target could be identified. Indeed, APS-1 constitutes a constellation of autoimmune diseases with public components (i.e. hypoparathyroidism, candidaiasis, and adrenal insufficiency) and a very extensive list of private components that can be severe or lifethreatening. These components are a challenge for the clinician but can be an opportunity to find new autoimmune components for the investigator. Finally, it remains to be evaluated whether more common form of bronchial manifestations have an autoimmune component and correspond to KCNRG autoimmunity.

References

1. Barry MJ: Screening for prostate cancer - the controversy that refuses to die. N Engl J Med 2009;360:1351-1354.

2. Brunet A: Cancer: when restriction is good. Nature 2009;458:713-714.

3. Holzenberger M: The role of insulin-like signalling in the regulation of ageing. Horm Res 2004;62(suppl 1):89-92.

4. Currie CJ, Poole CD, Gale EA: The influence of glucose-lowering therapies on cancer risk in type 2 diabetes. Diabetologia. 2009; [Epub ahead of print]. 